Research Article

\title{
Measurement and Uniform Formulation of Soil-Water Characteristic Curve for Compacted Loess Soil with Different Dry Densities
}

\author{
Yu Wang, ${ }^{1,2}$ Tonglu Li (D), $^{1,2}$ Ping Li, ${ }^{1,2}$ Yulu Lei, ${ }^{1,2}$ and David D. Lawrence ${ }^{1}$ \\ ${ }^{1}$ Department of Geological Engineering, Chang'an University, Xi'an 710054, China \\ ${ }^{2}$ Water Cycle and Geological Environment Observation and Research Station for the Chinese Loess Plateau, \\ Ministry of Education, Gansu 745399, China \\ Correspondence should be addressed to Tonglu Li; dcdgx08@chd.edu.cn
}

Received 4 December 2020; Revised 30 January 2021; Accepted 23 February 2021; Published 17 March 2021

Academic Editor: Jian Xu

Copyright (c) $2021 \mathrm{Yu}$ Wang et al. This is an open access article distributed under the Creative Commons Attribution License, which permits unrestricted use, distribution, and reproduction in any medium, provided the original work is properly cited.

To investigate the effect of dry density on the soil-water characteristics of compacted soil, loess used as filling in the land-making project of the Yan'an new district was collected and compacted to five initial dry densities of 1.40, 1.50, 1.60, 1.70, and 1.80 g/ $\mathrm{cm}^{3}$, respectively. The soil-water characteristic curves (SWCCs) of all specimens in the range of $0-10^{5} \mathrm{kPa}$ were measured using the filter paper method. The measured data were fitted using the Fredlund and Xing equation for each initial dry density. The SWCCs have obvious differences in a suction range below $100 \mathrm{kPa}$ and overlap when the suction range is higher. This suggests that the SWCC of compacted soil is independent of the initial dry density in the high suction range, but the correlation with the initial dry density exists in the low suction range. Therefore, the correlation functions of the parameters in the Fredlund and Xing equation with respect to the initial dry density were regressed, respectively. By substituting these functions into the Fredlund and Xing equation, the state surface function of $\theta_{w}-\psi-\rho_{d}$ was obtained and can reflect the SWCCs of all densities of the filled soil to support the further investigation of the unsaturated behavior of compacted soil.

\section{Introduction}

In the Chinese loess plateau, loess formations are used not only as natural foundation materials for buildings and infrastructures but also as compacted geomaterials for building foundations. With the rapid development of modern urban areas in the Chinese loess plateau in recent years, land-making projects, whose objective is to flatten hills and fill gullies to form a smooth spread of land for the development of urban sites or industrial plants, have been carried out for nearly a decade $[1,2]$. The largest such project is the new districts of Yan'an City, in the central region of China, as shown in Figure 1. Loess gullies are general V-shaped valleys; therefore, the thickness of the gully fillings is highly variable and can vary from a few meters up to $100 \mathrm{~m}$. The gully fillings comprise loess soil compacted in one layer with a thickness of several meters. The investigation has revealed that the fillings were a high heterogeneous soil for which the compactness is difficult to control according to the requirements of landmaking projects (Figure 2). Therefore, the prediction of the postfilling settlement and uneven settlement is a key issue for researchers, managers, and investors.

According to in situ monitoring data obtained at Yan'an new district, the main consolidation of the filled soil is accomplished just after the filling, and the postfilling settlement is minimal but continuous. The consolidation during filling is caused by the load of the overlaid soil. The filling is an unsaturated soil; however, surface water infiltration and groundwater penetration may change the soil-water characteristics and induce a large amount of postfilling settlement $[3,4]$. For unsaturated soil, the soil-water characteristic curve (SWCC) is important for investigating the soil behavior. Hence, this study focused on the measurement and uniformity of the SWCCs obtained for compacted loess soil with different dry densities. 

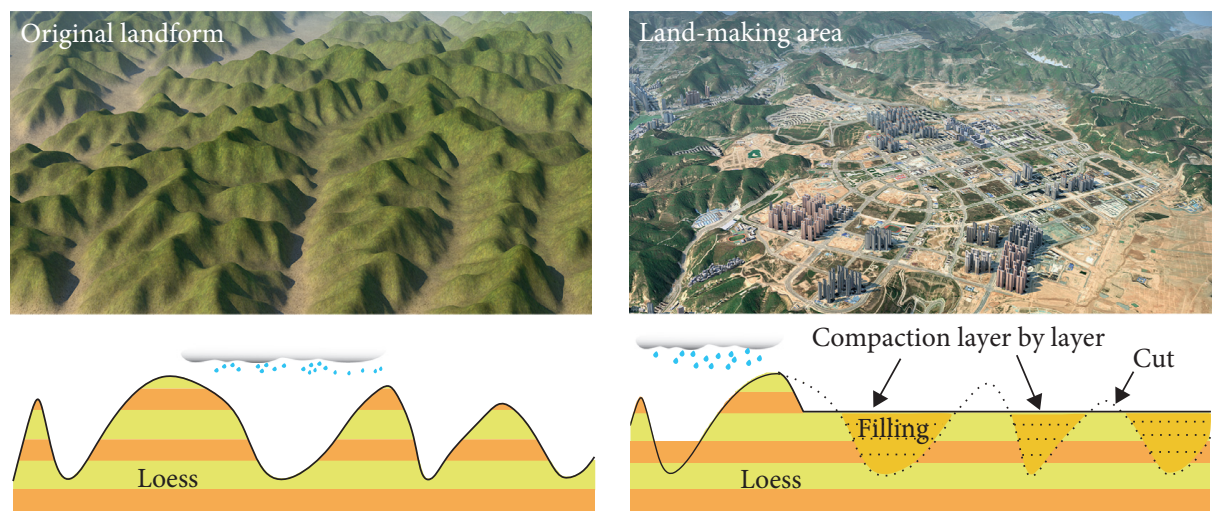

(a)

(b)

Figure 1: Overview of land-making area: (a) original landform; (b) after cutting and filling.

The SWCC describes the relationship between the matric suction and the gravimetric water content, volumetric water content, or saturation and plays a crucial role in modeling the behavior of unsaturated soils $[5,6]$. In fact, the measurement of the SWCC is the most important test required to introduce unsaturated soil mechanics into geotechnical engineering practice [7]. To date, the measurement of matric suction has been developed using the tensiometer method, axial translation technology (pressure plate instrument, Tempe instrument, centrifuge, etc.), and filter paper method [7]. Almost all suction measurement methods have shortcomings, including issues such as the range of application, cost, reliability, and feasibility [8].

Axial translation technology is a conventional method of measuring the soil matric suction in the range of $1-1.5 \mathrm{MPa}$. However, Baker and Frydman have argued that this method changes the energy state of the soil water by applying external pressure (up to $1 \mathrm{MPa}$ ) to the soil, which in turn changes the state and produces an unfavorable result compared with the natural field condition $(101.3 \mathrm{kPa})$ [9]. The commonly used tensiometer is limited within the scope of suction, that is, in the range of approximately $0-100 \mathrm{kPa}$ [10]. Various studies have shown that the filter paper method (FPM) is a relatively simple, low-cost, and time-efficient method that can be used to measure the suction in the range of $10-300,000 \mathrm{kPa}$ [11]. Since Hansen used blotting paper to determine the osmotic potential of sugar solutions at the same vapor pressure as the experimental soil specimens [12], the FPM has gradually been used to measure the soil-water potential. Additionally, Whatman No. 42 filter paper is widely used to measure soil suction [13-15] and is considered to be the most practical out of the three abovementioned methods. Moreover, this method does not affect the soil structure and can be used under atmospheric conditions with acceptable accuracy.

In this study, the fillings in the filled area of Yan'an new district were collected, and five groups of specimens with different initial dry densities in the range of the filled loess were generated using a static pressure instrument. The matric suction was measured using a modified FPM $\left(10-10^{5} \mathrm{kPa}\right)$ and the corresponding SWCCs were obtained.
The data measured for each specimen with different compacted density were fitted using the Fredlund and Xing equation [16] to obtain the respective SWCCs, and the SWCCs of the compacted loess were unified as a binary function of the matric suction against the water content and dry density, which can be used to investigate geotechnical problems related to compacted loess throughout the entire density and water content range.

\section{Soil Material for Tests}

The sampling site is located in the new district of Yan'an City, in the central region of China, where a new flat city site has been constructed by cutting and filling the hilly landscape (Figure 1). The deep gullies are filled by layered loess with a thickness of up to $100 \mathrm{~m}$, while the thickness of the cut section is up to $45 \mathrm{~m}$. According to the laboratory test data for 19 boreholes in the filled soil (Figure 2), the dry density of the compacted loess samples is mostly in the range of $1.40-1.80 \mathrm{~g} / \mathrm{cm}^{3}$ and accounts for $97 \%$ of the total density. This suggests that the dry density is far lower than the maximum value of $1.80 \mathrm{~g} / \mathrm{cm}^{3} \mathrm{ob}-$ tained by standard compaction in the laboratory and much lower than the required dry density of $1.70 \mathrm{~g} / \mathrm{cm}^{3}$. The water content of the vertical section exhibits great differences and tends to move away from the optimal value $(16 \%)$ in reality.

The filling was collected at the middle of the excavated profile to adequately represent the average components of compacted loess. The particle size, plastic limit, liquid limit, and specific gravity were measured first and the results are presented in Table 1. Additionally, as shown in Figure 3, the silt fraction $(0.002-0.05 \mathrm{~mm})$ makes up $66.0 \%$ of the total amount, and the clay particle $(<0.002 \mathrm{~mm})$ accounts for $6.6 \%$. The coefficient of uniformity is 8.5 and the coefficient of curvature is 1.4 , which is classified as well-graded cohesive soil $\left(C_{u}>5\right.$ and $\left.C_{C} \in[1,3]\right)$. According to the Unified Soil Classification System [17], the loess is classified as silty clay.

Previous studies have reported that the initial water content greatly influences the soil's pore structure [18]. 


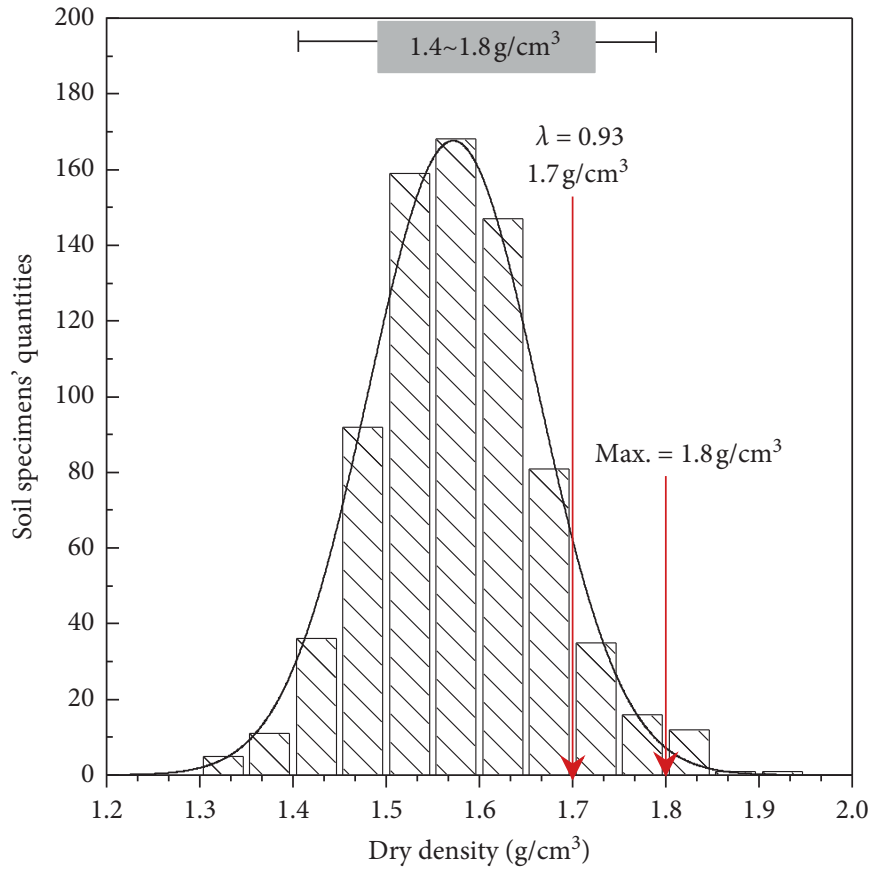

Distribution

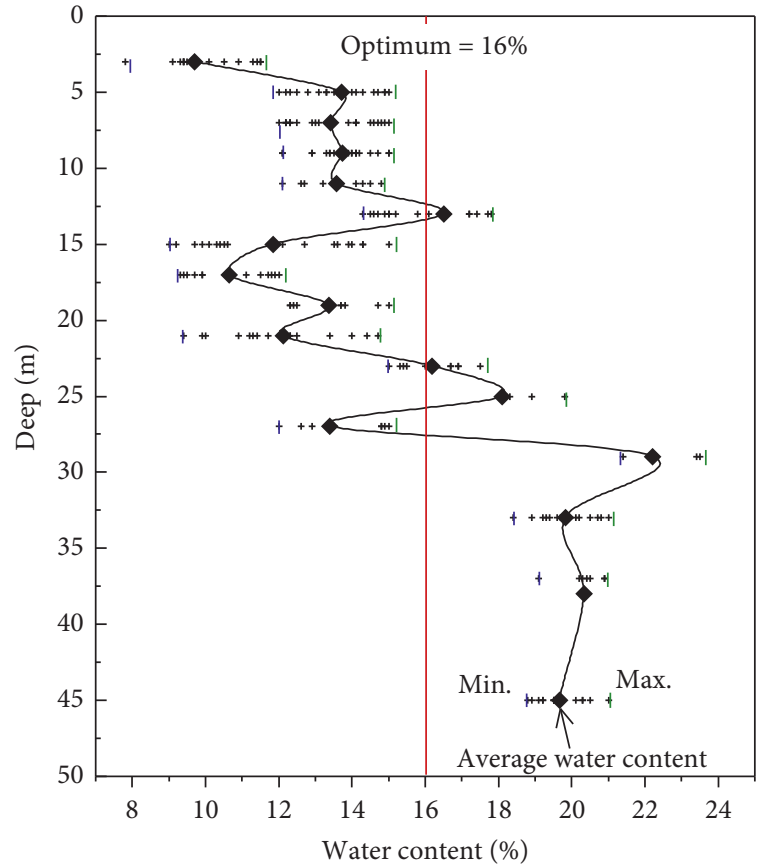

(b)

Figure 2: (a) Dry density and (b) water content in filled area.

TABle 1: Physical parameters of remolded specimens.

\begin{tabular}{lccccc}
\hline Specimen number & No. 1 & No. 2 & No. 3 & No. 4 & No. 5 \\
\hline Initial dry density $\left(\mathrm{g} / \mathrm{cm}^{3}\right)$ & 1.40 & 1.50 & 1.60 & 1.70 & 1.80 \\
Initial void ratio & 0.936 & 0.807 & 0.694 & 0.594 & 94 \\
Degree of compaction (\%) & 78 & 83 & 89 & 100 \\
Same parameters & Remolding water content & Specific gravity & Plastic limit & Liquid limit & Plasticity index \\
Value & $10 \%$ & 2.71 & $18.7 \%$ & $18.8 \%$ & 9.8 \\
\hline
\end{tabular}

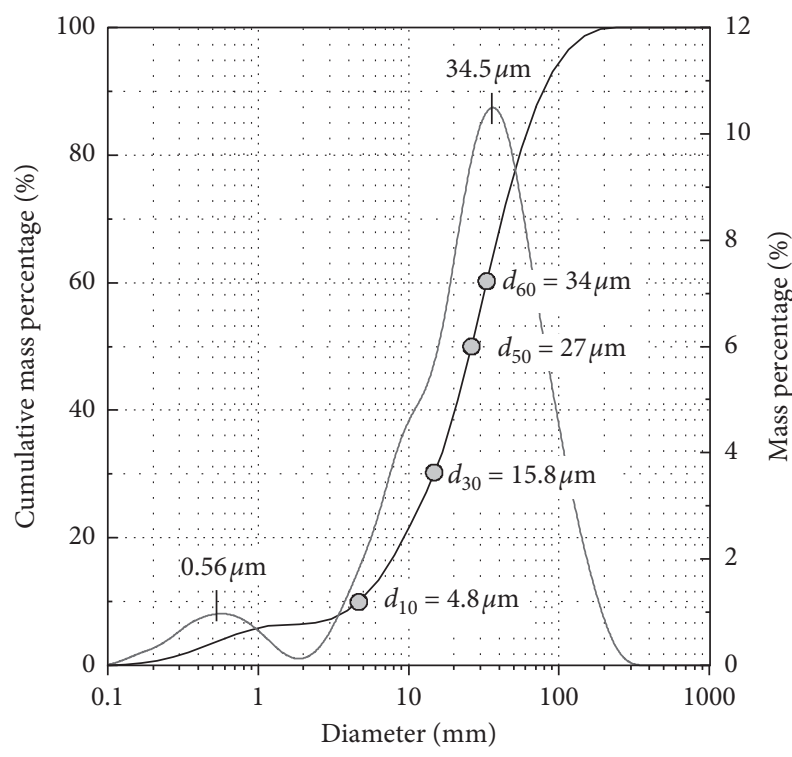

Figure 3: Curves of particle size distribution.
For compacted soil with the same void ratio, the macropores increase as the initial water content decreases [19]. However, there is a homogeneous distribution of pores in the compacted soil under the optimal water content [20]. For practical projects, the optimal water content is difficult to achieve and the water content is typically suboptimal, as shown in Figure 2. Therefore, to fully consider the soil-water characteristics of compacted soil with more macropores, the minimum average moisture content $(10 \%)$ in the filling area was selected for compaction.

Subsequently, the filling was crushed and remolded into five groups based on the results of their initial dry densities. The target initial dry densities are 1.40, 150, $1.60,1.70$, and $1.80 \mathrm{~g} / \mathrm{cm}^{3}$ and cover $97.0 \%$ of the filled loess specimens at the site. The corresponding void ratio and bulk density are listed in Table 1 . The water content of all remolded specimens was controlled to be $10 \%$, and the initial dry density error was controlled to be $\pm 0.01 \mathrm{~g} / \mathrm{cm}^{3}$. 


\section{Method of Measuring SWCC}

The principle of the FPM can be understood as the transportation of the pore water in the soil to wet or dry filter paper. The matric suction is measured as the soil specimen contacts the filter paper. And when there is no direct contact between them, the total suction (equal to the sum of the matric suction and osmotic suction) is also measured $[6,21]$. In this study, the contact method was used to measure the matric suction, and the oven-drying method was used to measure the water content.

Once the specimens were remolded, they were put into sealed desiccators to maintain the original water content and keep free from disturbances. The specimens for each initial dry density were set as a group and used to measure the SWCC. The measuring processes were carried out as follows:

(1) Filter paper preparation: ordinary filter paper was cut to the same size as the specimen surface with a diameter of $61.8 \mathrm{~mm}$ (equal to the size of the specimens), as shown in Figure 4(a), and used to cover the surface of the specimens to protect them against falling slags and to protect the test filter paper against contamination. The test filter paper was slightly smaller than the specimen or ordinary filter paper and had a diameter of $42.5 \mathrm{~mm}$. Both the ordinary filter paper and the measuring filter paper were soaked in formalin solution (2\%) and then dried to prevent the growth of microorganisms before use [22]. Notably, the weighing of the filter paper was carried out in a clean aluminum box with a cover.

(2) Specimens preparation: first, using a static compression method at the water content of $10 \%$, the specimens were remolded with a definite initial dry density in metal rings with a diameter of $61.8 \mathrm{~mm}$ and height of $20 \mathrm{~mm}$. Subsequently, the set of specimens were dried in an oven at $120^{\circ} \mathrm{C}$ for 24 hours. This drying method may cause changes to the soil microstructure [23, 24], and freeze-drying is probably the most appropriate way of avoiding this issue when testing the wetting SWCC $[25,26]$. Next, water was dropped on the specimens to control the target water content from very low (2\%) to saturated at an increasing interval of $1 \%$ or $2 \%$. Two parallel specimens with the same water content were prepared.

(3) Water balance in the specimens: as soon as water was dropped on a specimen, the process of wrapping the specimen began, as shown in Figure 4. Specimens with varying water content were placed into the moisturizer for 48 hours and were packed after being removed. First, the initial weight of the soil specimens and filter paper was measured, and then the filter paper was nimbly placed between two identical specimens, as shown in Figure 4(b). Subsequently, the upper and lower specimens were fixed using waterproof tape and tightly wrapped up using plastic wrap to create a simple waterproof environment, as shown in Figure 4(c). Aluminum foil paper was used as a protective and shaping material to further wrap the specimens [27-29], and melted paraffin wax was evenly brushed on the aluminum foil paper such that the entire specimen was sealed with wax, as shown in Figure 4(d). Finally, the specimens were placed in the incubator $\left(20^{\circ} \mathrm{C}\right)$ for soil-water balancing after being labeled.

(4) Weighing of filter paper and specimens: the specimens were removed after seven days of balancing in the incubator $[9,22]$. After unpacking the wrapped specimens, the mass of the wet soil specimens and the wet test filter paper were weighed, respectively, and both were dried and then weighed again. Notably, the filter paper must be quickly put into an aluminum box using tweezers and the box should be covered for weighing, which is a key operation to ensure the accuracy of the data. In this process, an ordinary balance (accuracy of $0.01 \mathrm{~g}$ ) was used to weigh the soil specimens and the Mettler analytical balance (accuracy of $0.0001 \mathrm{~g}$ ) was used to weigh the filter paper, owing to the different precision requirements, as shown in Figure 5. Moreover, different ovens were used to avoid contamination.

(5) Calculating the matric suction and water content of the specimens: according to the above-mentioned operation, the weight of the test filter paper before and after balancing can be obtained for each specimen, and the matric suction can be calculated using the calibration equation (1) [22].

$$
\begin{aligned}
& \log _{10} \psi=5.327-0.0779 * w_{f p}\left(w_{f p}<45.26 \%\right), \\
& \log _{10} \psi=2.412-0.0135 * w_{f p}\left(w_{f p}>45.26 \%\right),
\end{aligned}
$$

where $\psi$ represents the matric suction $(\mathrm{kPa}) ; w_{f p}$ represents the gravity water content change of the filter paper (\%).

\section{Regression for SWCCs with Measured Data}

Among the many empirical models (such as Gardner, Brooks and Corey, van Genuchten, McKee, and Bumb), the Fredlund et al. model [16] is widely used throughout the entire suction range (equation (2)) and has a correction factor $c(\psi)$ (equation (3)) that always makes the suction under zero water content approach $10^{6} \mathrm{kPa}$. In equation (2), parameter $a$ is the air entry value of soil; $n$ is the inflection rate in the transient zone, and as this value increases, the inflection rate also increases, which reflects the pore size uniformity; $m$ controls the shape in the low suction range.

$$
\begin{aligned}
\theta_{w} & =c(\psi) \frac{\theta_{s}}{\left[\ln \left(e+(\psi / a)^{n}\right)\right]^{m}}, \\
c(\psi) & =1-\frac{\ln \left(1+\left(\psi / \psi_{r}\right)\right)}{\ln \left(1+\left(10^{6} / \psi_{r}\right)\right)},
\end{aligned}
$$

where $\theta_{w}$ is the gravity water content $(\%) ; \theta_{s}$ is the saturated gravity water content $(\%) ; \psi$ is the matric suction $(\mathrm{kPa})$; $e \approx 2.71828$. 


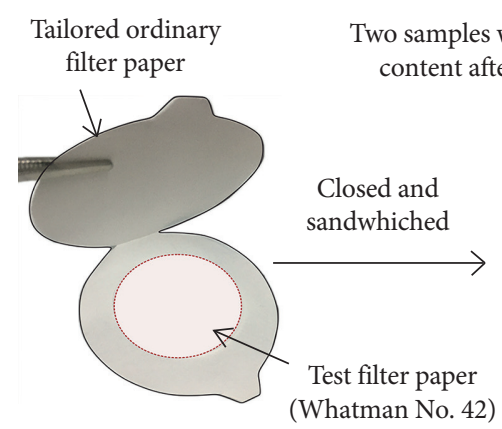

(a)
(Whatman No. 42)

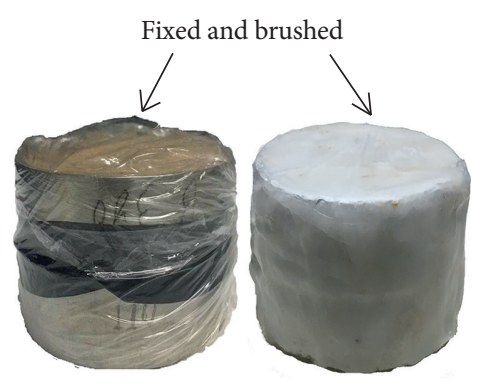

(c)

(d)

FIgure 4: Process of wrapping specimens.

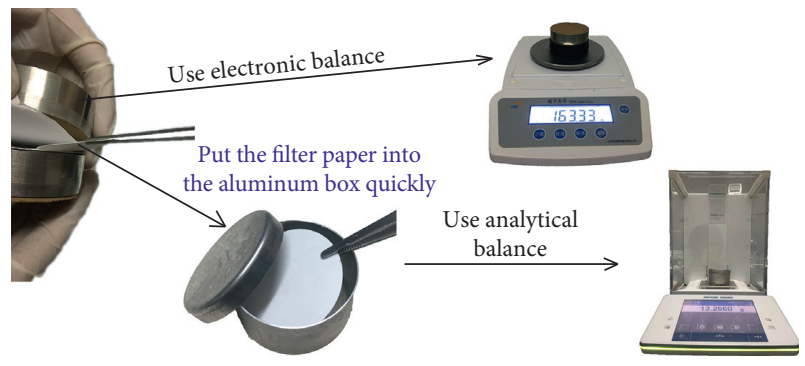

Figure 5: Process of removing specimens.

As shown in Figure 6, the fitting curves intersect at approximately $100 \mathrm{kPa}$ using equation (2), and the data measured for different initial dry densities overlap in a suction range greater than $100 \mathrm{kPa}$, but the curves are more scattered. This suggests that the SWCCs in this range are independent of the initial dry density and exhibit a linear relationship in the semilogarithmic coordinate system. Other studies have also confirmed that the curves for the same type of soil exhibit similar distribution characteristics under different pressures [9], which is called the "broom" shape [30-31]. Therefore, it is reasonable to use piecewise fitting. Equation (2) is used for the range of $1-100 \mathrm{kPa}$, and linear fitting is used over $100 \mathrm{kPa}$. All measured data obtained in the range of $1-100 \mathrm{kPa}$ were fitted using equation (2). The parameters are listed in Table 2 and the fitting curves are shown in Figure 7.

As shown in Figure 7, according to the shape and physical context of the curves, each SWCC can be divided into three stages in the wetting curve: the residual stage, transient stage, and boundary effect stage [5]. During the wetting process, the transient stage and boundary stage are divided by the air occlusion value (AOV), while the transient stage and residual stage are divided by the residual value (RV). The AOV and RV of the SWCC can be determined using the method proposed by Vanapalli et al. [5], and the data are also presented in Table 2.

In the wetting process, the first stage is the residual stage. The water molecules are adsorbed by the soil particles to form adsorbed water, and the pore space is essentially occupied by the gas phase as the suction increases. The water content of the soil slowly changes, the performance tends to be consistent, and the RVs of all specimens are approximately $100 \mathrm{kPa}$ with a water content of approximately $11 \%$.

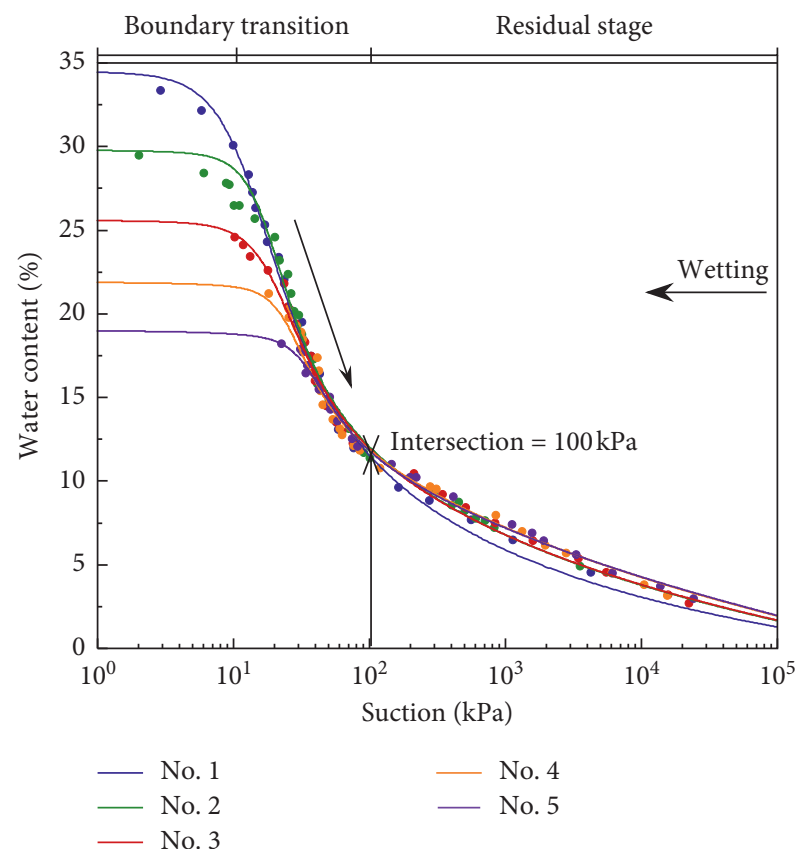

Figure 6: Test data and fitting curves of five specimens using Fredlund et al. [16] model.

During the transient stage, capillarity becomes dominant and the water begins to occupy the smallest pore in the soil, which leads to the escape of air. Thus, the soil is in a three-phase state (solid, liquid, and air), which is referred to as the contractile skim or interface by Fredlund [32]. Most engineering problems related to unsaturated soils occur at this stage. As the water content increases, the suction starts to decrease in a rapid manner. The change at this stage is closely associated with the initial dry density and the path is positively correlated with the initial dry density.

When the suction exceeds the AOV, the soil enters the boundary stage and is nearly saturated. Additionally, the water phase is continuous and the gas phase is suspended in the water in the form of closed bubbles. As can be seen from the curves, specimen No.1 $\left(1.4 \mathrm{~g} / \mathrm{cm}^{3}\right)$ has an AOV of $5 \mathrm{kPa}$ and corresponding water content of $33 \%$, while No. 5 (1.8 g/ $\mathrm{cm}^{3}$ ) has the highest AOV of $11.5 \mathrm{kPa}$ corresponding to a water content of $18 \%$. 
TABLE 2: Fitting parameters.

\begin{tabular}{lccccc}
\hline Specimen number & No. 1 & No. 2 & No. 3 & No. 4 & No. 5 \\
\hline Initial dry density $\left(\mathrm{g} / \mathrm{cm}^{3}\right)$ & 1.4 & 1.5 & 1.6 & 1.7 & 1.8 \\
\hline$A$ & 11.47 & 14.53 & 18.026 & 24.584 & 32.304 \\
$N$ & 1.71 & 2.356 & 3.361 & 4.93 & 5.930 \\
$M$ & 0.765 & 0.550 & 0.402 & 0.283 & 0.213 \\
$R^{2}$ & 0.98 & 0.98 & 0.98 & 0.95 & 0.99 \\
$\theta_{s}(\%)$ & 34.5 & 29.8 & 25.6 & 21.9 & 18.7 \\
AOV $(\mathrm{kPa})$ & 5 & 9 & 10.2 & 11 & 11.5 \\
$\mathrm{RV}(\mathrm{kPa})$ & 100 & 100 & 100 & 100 & 100
\end{tabular}

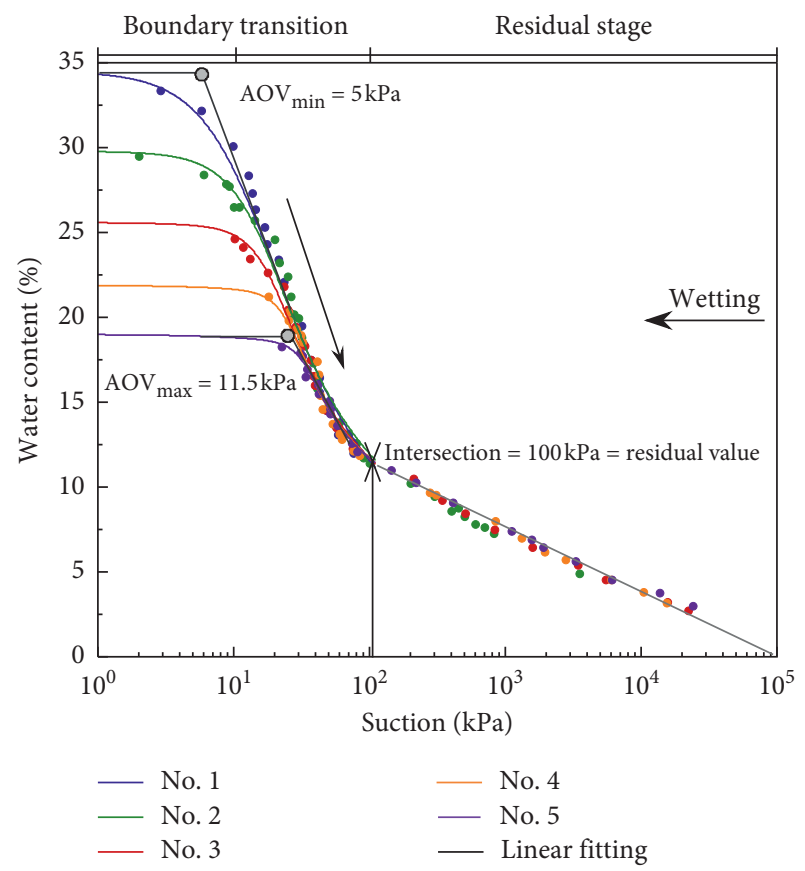

Figure 7: Test data and fitting curves of five specimens using piecewise fitting.

The Equi-suction lines (Figure 8) also show that the lines below $100 \mathrm{kPa}$ have a high inclination degree and the specimens with lower initial dry density have a higher moisture content under the same suction condition. The lines above $100 \mathrm{kPa}$ are considered to be approximately vertical, which means that the initial dry density or pore ratio of the compacted soil in the high suction phase is independent of the suction. According to Ridley and Delage (1998), Delage (2007), and Rafael and Bake (2009), within the scope of dominant adsorption, suction should only depend on the gravity water content of remolded clay, and there is no correlation between the dry density or pore ratio

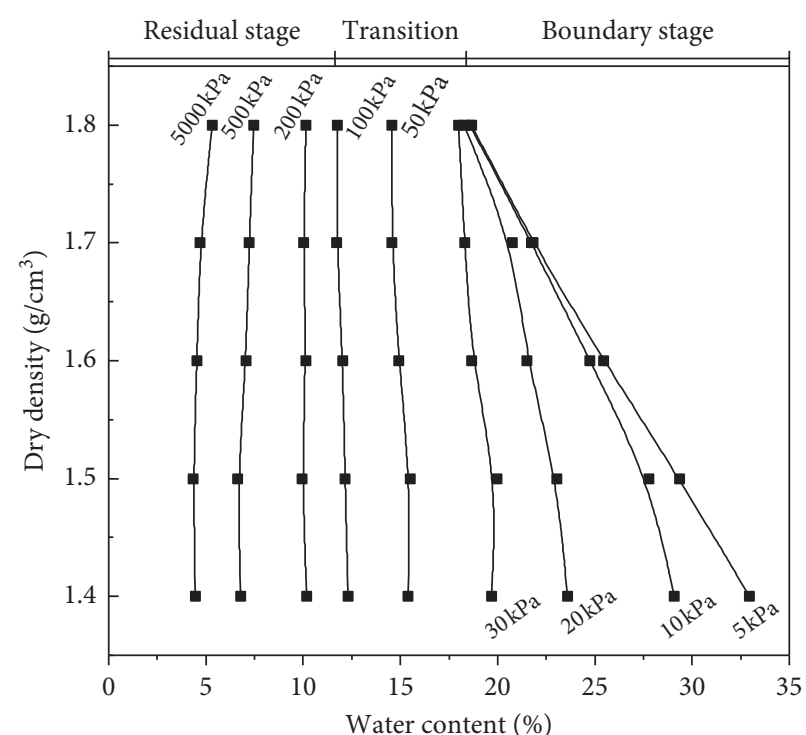

Figure 8: Equi-suction lines of specimens with different initial dry density.

of the soil at the high suction phase and suction change $[9,33,34]$.

Generally, as the initial dry density increases, the path of the transient stage becomes shorter while that of the boundary stage becomes longer, but the difference of the residual region is not obvious.

\section{Unified SWCC of Compacted Loess}

By plotting the values of the fitting parameters $a, m$, and $n$ listed in Table 2 against the initial dry density shown in Figure 9, it can be seen that each of them has a good correlation with the initial dry density. The functions of the parameters versus the initial dry density were also obtained by regression analysis, and $a$ is exponentially correlated with the initial dry density, as expressed by equation (4), while $n$ and $m$ are linearly correlated with the initial dry density, as expressed by equations (5) and (6). Additionally, there exists a linear relationship between the saturated water content and the initial dry density (equation (7)), and we consider that the matric suction in the residual stage tends to be consistent. Therefore, it is reasonable to consider that all specimens have the same residual matric suction, which is approximately equal to $100 \mathrm{kPa}$. By substituting these functions into the Fredlund and Xing equation, the united function (equation (8)) can be determined and used as an empirical equation reflecting a series of the SWCC.

$$
\begin{aligned}
& a=0.255 e^{2.688 \rho_{d},} \\
& n=11.014 \rho_{d}-13.695, \\
& m=-1.371 \rho_{d}-2.636,
\end{aligned}
$$




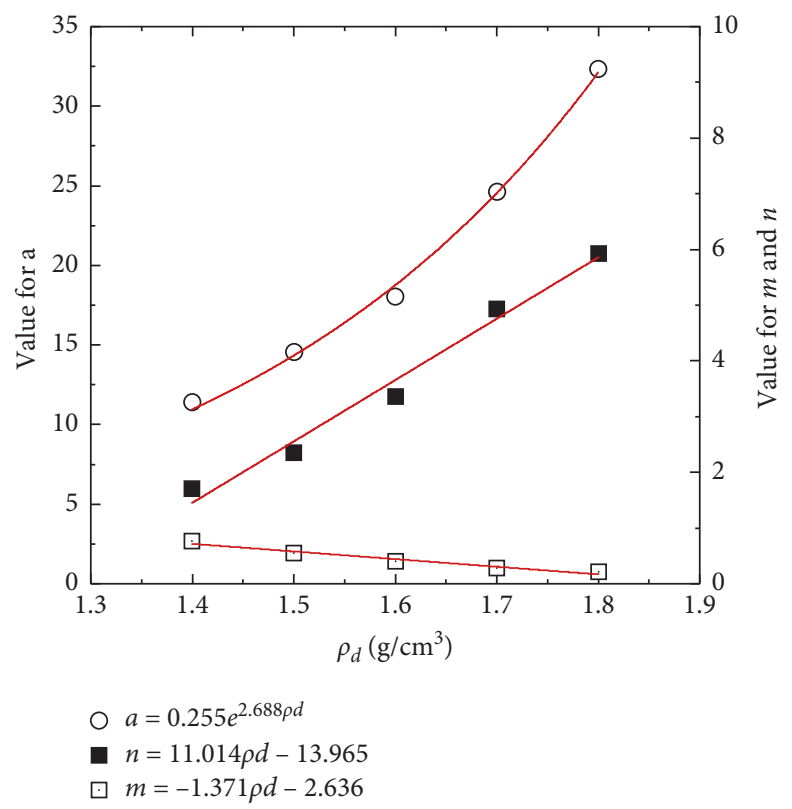

FIgURE 9: Relationship between $a, n$, and $m$ and initial dry density.

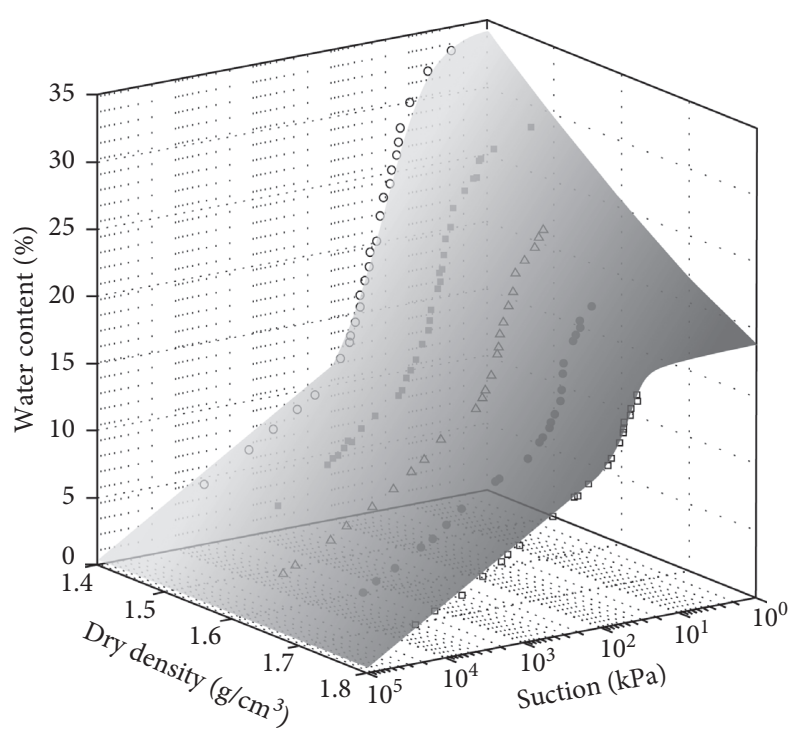

Figure 10: Unified function of $\theta_{w}-\psi-\rho_{d}$.

$$
\begin{aligned}
\theta_{s} & =-39.5 \rho_{\mathrm{d}}+89.3, \\
\theta_{w} & =c(\psi) \frac{\theta_{s}}{\left\{\ln \left(e+\left[\psi / 0.255 e^{\left(2.688 \rho_{d}\right)}\right]^{\left(11.014 \rho_{d}-13.695\right)}\right\}^{1.371 \rho_{d}+2.636}\right.}, \quad(\psi<100), \\
\theta_{w} & =-3.707 \log \psi+19.02, \quad(\psi \geq 100),
\end{aligned}
$$

where $\theta_{w}$ is the gravity water content (\%); $\theta_{s}$ is the saturated gravity water content $(\%) ; \psi$ is the matric suction $(\mathrm{kPa}) ; \psi_{r}$ is the residual matric suction $(\mathrm{kPa}) ; e \approx 2.7$.
Figure 10 shows the fitting surface $\left(\theta_{w}-\psi-\rho_{d}\right)$, and the five sets of experimental data are in good agreement. It can be intuitively understood that, on the surface, the boundary 
effect area is enlarged, the transient area is reduced (slope becomes steeper), and the residual area remains the same as the initial dry density increases. Using equation (8), as long as the dry density is given, a corresponding SWCC can be determined on this fitting surface.

\section{Summary and Conclusion}

This study measured the SWCCs of compacted loess soil with different density using the FPM, and a unified function based on the Fredlund and Xing equation was regressed. It is expected that the findings of this study will provide the foundation for further research on unsaturated soil.

(1) In this paper, the steps for measuring the SWCC using the contact FPM are described in detail. With this procedure, the SWCC in the range of $1-10,000 \mathrm{kPa}$, or even higher, can be determined quickly and more accurately. Thus, this method should be widely adopted.

(2) The influence of the initial dry density on the soilwater retention behavior was analyzed. The SWCCs overlapped when the moisture content was less than $11 \%$, which corresponds to a suction above approximately $100 \mathrm{kPa}$. For the same type of soil, compaction only changed the soil-water retention behavior when the suction was less than $100 \mathrm{kPa}$, and the retention capacity decreased as the compactness increased, whereas the soil-water characteristics essentially remained the same when the suction was greater than $100 \mathrm{kPa}$.

(3) A "nonlinear + linear" piecewise fitting method was used to fit the measuring data. The equation $\left(\theta_{w}-\psi-\rho_{d}\right)$ reflecting the SWCCs of the entire filling area was obtained through regression analysis, which is significant for practical engineering. Additionally, this is the main equation used to calculate the seepage field change and settlement under infiltration conditions in the filling area.

\section{Data Availability}

The data used to support the findings of this study are available from the corresponding author upon request.

\section{Conflicts of Interest}

The authors declare that there are no conflicts of interest regarding the publication of this paper.

\section{Acknowledgments}

The study was supported by the National Natural Science Foundation of China (Grant nos. 41772278, 41877242, 42072311, and 41790442) and the Fundamental Research Funds for the Central Universities, CHD (300102260204). The above financial supports are gratefully acknowledged.

\section{References}

[1] P. Li, H. Qian, and J. Wu, "Environment: accelerate research on land creation," Nature, vol. 510, no. 7503, pp. 29-31, 2014.

[2] X. Pan, Y. Li, Q. Yu, X. Shi, D. Yang, and K. Roth, "Effects of stratified active layers on high-altitude permafrost warming: a case study on the Qinghai-Tibet Plateau," The Cryosphere, vol. 10, no. 4, pp. 1591-1603, 2016.

[3] M. Hino, Y. Odaka, K. Nadaoka, and A. Sato, "Effect of initial soil moisture content on the vertical infiltration process-a guide to the problem of runoff-ratio and loss," Journal of Hydrology, vol. 102, no. 1-4, pp. 267-284, 1988.

[4] C. Yang, D. Sheng, and J. P. Carter, "Effect of hydraulic hysteresis on seepage analysis for unsaturated soils," Computers and Geotechnics, vol. 41, pp. 36-56, 2012.

[5] S. K. Vanapalli, D. G. Fredlund, and D. E. Pufahl, "Influence of soil structure and stress history on the soil-water characteristics of a compacted till," Géotechnique, vol. 51, no. 6, pp. 573-576, 2001.

[6] L. Ning and W. J. Likos, Unsaturated Soil Mechanics, Wiley Publications, Hoboken, NJ, USA, 2004.

[7] N. Lu and W. J. Likos, "Suction stress characteristic curve for unsaturated soil," Journal of Geotechnical and Geoenvironmental Engineering, vol. 132, no. 2, pp. 131-142, 2006.

[8] R. Bulut and E. C. Leong, "Indirect measurement of suction," Geotechnical and Geological Engineering, vol. 26, no. 6, pp. 633-644, 2008.

[9] R. Baker and S. Frydman, "Unsaturated soil mechanics: critical review of physical foundations," Engineering Geology, vol. 106, no. 1-2, pp. 26-39, 2009.

[10] F. Masrouri, K. V. Bicalho, and K. Kawai, "Laboratory hydraulic testing in unsaturated soils," Geotechnical and Geological Engineering, vol. 26, no. 6, pp. 691-704, 2008.

[11] F. A. M. Marinho and O. M. Oliveira, "The filter paper method revisited," Geotechnical Testing Journal, vol. 29, no. 3, pp. 250-258, 2006.

[12] H. C. Hansen, "The water-retaining power of the soil," The Journal of Ecology, vol. 14, no. 1, pp. 111-119, 1926.

[13] R. Ammavajjala, B. B. Munwar, and A. A. B. Moghal, "Effect of fines content on the hysteretic behavior of water retention characteristic curves of reconstituted soils," Journal of $M a$ terials in Civil Engineering, vol. 32, no. 4, 2020.

[14] H. Li, T. L. Li, R. J. Jiang, Y. Wang, and Y. G. Zhang, “A new method to simultaneously measure the soil-water characteristic curve and hydraulic conductivity function using filter paper," Geotechnical Testing Journal, vol. 43, no. 6, pp. 1541-1551, 2020.

[15] A. Pedarla, A. J. Puppala, U. D. Patil, L. R. Hoyos, and A. H. Pino, "A semi-empirical approach-based model for swell characterization of expansive clays," Geotechnical and Geological Engineering, vol. 37, no. 6, pp. 5371-5381, 2019.

[16] D. G. Fredlund, A. Xing, and S. Huang, "Predicting the permeability function for unsaturated soils using the soilwater characteristic curve," International Journal of Rock Mechanics \& Mining Ence \& Geomechanics Abstracts, vol. 32, no. 4, p. 159A, 1994.

[17] A. International, Standard Practice for Classification of Soils for Engineering Purposes (Unified Soil Classification System), Astm, West Conshohocken, PA, USA, 2006.

[18] J. D. Mckinley, E. J. Murray, W. C. Tan et al., "Wetting, drying and compression characteristics of compacted clay," Géotechnique, vol. 56, no. 1, pp. 57-62, 2006.

[19] J. J. Wang, H. Y. Zhou, and t. X. Wang, "Experimental study on structural characteristics of unsaturated compacted loess," 
Geotechnical Mechanics, vol. 40, no. 4, pp. 1351-1357, 2019, in Chinese.

[20] Y. Z. Hao, T. H. Wang, and J. J. Wang, "Structural properties of unsaturated compacted loess for various sample moisture contents," Arabian Journal of Geosciences, vol. 12, no. 8, 2019.

[21] S. Al-Khafaf and R. J. Hanks, "Evaluation of the filter paper method for estimating soil water potential," Soil Science, vol. 117, no. 4, pp. 194-199, 1974.

[22] ASTM, Standard Test Method for Measurement of Soil Potential (Suction) Using Filter Paper, American Society for Testing and Materials, West Conshohocken, PA, USA, 2011.

[23] J. A. Staricka and G. R. Benoit, "Freeze-drying effects on wet and dry soil aggregate stability," Soil Science Society of America Journal, vol. 59, no. 1, pp. 218-223, 1995.

[24] L. J. Deacon, D. V. Grinev, J. W. Crawford, J. Harris, K. Ritz, and I. M. Young, "Simultaneous preservation of soil structural properties and phospholipid proflles: a comparison ofThree drying techniques," Pedosphere, vol. 18, no. 3, pp. 284-287, 2008.

[25] P. Zhang, Y. Fang, X. Yan, and Z. He, "Study of different dry methods for drying remolded bentonite sample with mercury intrusion test," Geotechnical Mechanics, vol. 32, no. S1, pp. 388-391, 2011, in Chinese.

[26] D. Dagesse, "Effect of freeze-drying on soil aggregate stability," Soil Science Society of America Journal, vol. 75, no. 6, pp. 2111-2121, 2011.

[27] X. Li, "Experimental exploration of metal composition in tin paper," Chemistry Teaching, no. 9, pp. 80-82, 2018, in Chinese.

[28] R. Ranau, J. Oehlenschläger, and H. Steinhart, "Aluminium levels of fish fillets baked and grilled in aluminium foil," Food Chemistry, vol. 73, no. 1, pp. 1-6, 2001.

[29] M. Lamberti and F. Escher, "Aluminium foil as a food packaging material in comparison with other materials," Food Reviews International, vol. 23, no. 4, pp. 407-433, 2007.

[30] G. Tao, J. Zhang, X. Zhuang, and L. Yang, "Influence of compression deformation on the soil-water characteristic curve and its simplified representation method," Journal of Hydraulic Engineering, vol. 45, no. 10, pp. 1239-1246, 2014, in Chinese.

[31] G. Tao and L. Kong, "Prediction of air-entry value and soilwater characteristic curve of soils with different initial void ratios," Chinese Journal of Geotechnical Engineering, vol. 40, no. S1, pp. 34-38, 2018, in Chinese.

[32] G. F. Delwyn, "Unsaturated soil mechanics in engineering practice," Journal of Geotechnical and Geoenvironmental Engineering, vol. 132, no. 3, 2006.

[33] P. Delage, "Microstructure features in the behavior of engineered barriers for nuclear waste disposal. Experimental unsaturated soil mechanics," in Proceedings in Physics, vol. 112, pp. 11-32, 2007.

[34] A. M. Ridley and J. Perez-Romero, "Suction-water content relationships for a range of compacted clays," in Proceedings of 2nd International Conference on Unsaturated Soils, Beijing, China, August 1998. 